\title{
Estudio para la optimización de mallas estructurales de acero envolventes de edificios en altura según sus solicitaciones, en base al análisis de sus líneas isostáticas
}

\author{
Study to optimize steel space grid envelope structures for tall buildings \\ according to their solicitations, based on their isostatic lines
}

$\underline{\text { R. Señís }}^{(*)}$, R. Sastre ${ }^{(*)}$, R. Brufau ${ }^{(*)}$, E. C. Carbajal ${ }^{(*)}$

RESUMEN

El objetivo del presente estudio es determinar si las mallas estructurales de acero, envolventes de edificios en altura (entramado en tubo), son más óptimas y eficientes cuando su diseño se adapta a la trayectoria de las líneas isostáticas de los elementos estructurales equivalentes bajo las acciones solicitantes. Se analizan 600 modelos de edificios en altura, para distintas triangulaciones de fachada y esbelteces. Las cargas de viento que influyen sobre los edificios se determinan con el método de Cargas Estáticas Equivalentes de acuerdo a la velocidad del viento y turbulencia provocados por la rugosidad del terreno y la topografía. También será necesario determinar las frecuencias y modos de vibración propios de cada estructura, así como definir los coeficientes de presión, de fuerzas y de momentos resultantes del viento. Los resultados concluyen que las mallas ganan eficiencia cuando identifican la trayectoria de las líneas isostáticas generadas por la acción principal, el viento.

Palabras claves: Edificios en altura; mallas espaciales; entramado en tubo; líneas isostáticas; cargas estáticas equivalentes de viento.

\section{ABSTRACT}

The aim of the present study is determinate if the steel space grid envelope structures, for tall buildings (framed tube), are more optimal and efficient if their design is based on the isostatic lines of their equivalents structural elements according to their solicitations. In this study, 600 models of tall buildings, for different triangled façades and geometric slenderness, are analyzed. The methodology employed consisted of detecting the wind loads that affects tall buildings applying the Equivalent Static Wind Loads (ESWL) in relation to the wind speed and turbulence result of the roughness terrain and the topographic. However, it was also needed to establish the frequencies and modes of vibration of each structure as well as defining the coefficients of pressure, force and moments produced by the wind. The results conclude that space grids are more efficient if they do adopt the trajectory of the main and dominant load, in this case the wind.

Keywords: Tall buildings; space grid structures; framed tube; isostatic lines; equivalent static wind loads.

\footnotetext{
(*) Escuela Politécnica Superior de Edificación de Barcelona UPC-BarcelonaTech, Barcelona (España).

(**) Escuela Técnica Superior de Arquitectura del Vallés UPC-BarcelonaTech, Sant Cugat del Vallès (España). Persona de contacto/Corresponding author: roger.senis@upc.edu (R. Señís)
}

Cómo citar este artículo/Citation: Señís, R., Sastre, R., Brufau, R., Carbajal, E.C. (2014). Estudio para la optimización de mallas estructurales de acero envolventes de edificios en altura según sus solicitaciones, en base al análisis de sus líneas isostáticas. Informes de la Construcción, 66(EXTRA-1): moo5, doi: http://dx.doi.org/10.3989/ic.13.094.

Licencia / License: Salvo indicación contraria, todos los contenidos de la edición electrónica de Informes de la Construcción se distribuyen bajo una licencia de uso y distribución Creative Commons Reconocimiento no Comercial 3.o. España (cc-by-nc). 


\section{INTRODUCCIÓN}

\subsection{Líneas isostáticas}

Resulta relevante el estudio de las líneas isostáticas de un elemento, entendidas como las envolventes de las direcciones principales de todos sus puntos, en el diseño y análisis de estructuras arquitectónicas, para un mejor comportamiento y en definitiva su optimización según la forma y sus solicitaciones. En la Figura 1, se puede observar que trazado tienen las líneas isostáticas del módulo tipo de la cubierta del Hipódromo de la Zarzuela en Madrid, obra del ingeniero de caminos Eduardo Torroja en el año 1935. Tal y como se observa, el estudio de las líneas isostáticas del elemento estructural permite definir la distribución del armado pasivo de la losa de hormigón, mejorando su eficacia y el comportamiento del elemento estructural. Concepto que el maestro Eduardo Torroja define como:

«La obra mejor es la que se sostiene por su forma y no por la resistencia oculta de su material», -Fernández, et al. (1)-.
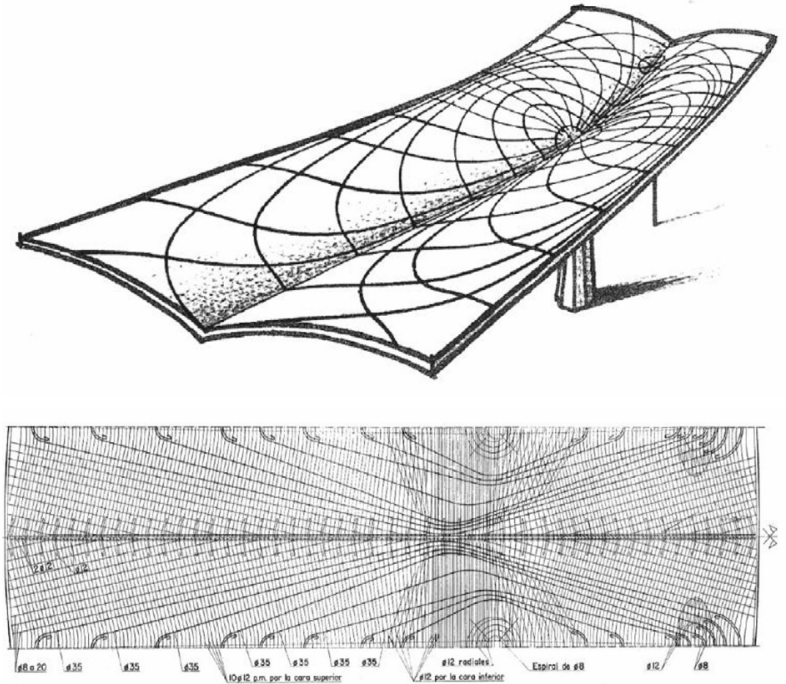

Figura 1. Estructura laminar cubierta Hipódromo de la Zarzuela de Madrid (1935) obra de E. Torroja, -Fernández, et al. (1)-

Son muchos los proyectos que se basan en este concepto estructural para mejorar su comportamiento. Obras con nervaduras de Pier Luigi Nervi, obras de Antonio Gaudí con formas basadas en la naturaleza, los proyectos de Cecil Balmond, Robert Maillart, Félix Candela o Mutsuro Sasaki, son algunos ejemplos. Por ello, son muchos los estudios realizados sobre el comportamiento estructural de acuerdo a las direcciones principales, y en definitiva, según las líneas isostáticas de los elementos estructurales. Algunos de estos estudios han sido realizados por autores como D'A. Thompson (2), J. E. Gordon (3), M. Salvadori, et al. (4), F. Moussavi (5), A. Muttoni (6) o M. Mimram (7) a propósito de la obra de Le Ricolais.

También se puede observar la función que desempeña la forma del elemento estructural, en este caso, la curvatura de la cáscara de hormigón le da una mayor rigidez y capacidad resistente, como cuando se dobla una hoja de papel para tener una mayor resistencia y estabilidad respecto a la hoja sin doblar. Concepto que Salvadori, et al. (4), define como:

«La misma hoja de papel, doblada en un punto y con una ligera curvatura hacia arriba, soporta su peso propio y aun alguna carga adicional [...]. La nueva capacidad portante no se obtiene aumentando la cantidad de material, sino dándole forma adecuada».

Existe un número significativo de edificios arquitectónicos que se han basado en este concepto para optimizar su diseño y su comportamiento estructural, siendo de aplicación marcando unas pautas para el diseño y el análisis de mallas estructurales envolventes de edificios en altura.

\subsection{Edificios en altura}

En 1885, después del gran incendio de la ciudad de Chicago (1871), se construyó el que se conoce como el primer rascacielos del mundo con estructura metálica, -Larson (8)-. Esta construcción fue posible gracias al invento de Elisha G. Otis en el año 1854: el ascensor con autofrenado -Gómez-Hermoso, et al. (9)-. Un invento que va íntimamente relacionado con los edificios en altura, ya que éstos, no se podrían concebir sin él. El edificio de 10 plantas y $42 \mathrm{~m}$ de altura conocido como Home Insurance Building de Chicago, obra de William Le Baron Jenney, -Larson (8)-, fue el inicio de un cambio en la tipología estructural de los edificios para posibilitar la evolución futura.

Desde entonces, son muchos los edificios que han buscado crecer en altura como símbolo de poder y avance tecnológico. Edificios como el Burj Khalifa en Dubai o el emblemático Empire States de 380 m, -Holmes (10)-, son un claro reflejo de esta idea. Por ello, son muchos los estudios, como por ejemplo los realizados por Fazlur R. Khan (11), sobre esta tipología estructural (Figura 2) y sobre la adaptación de cada modelo más adecuado para su altura.

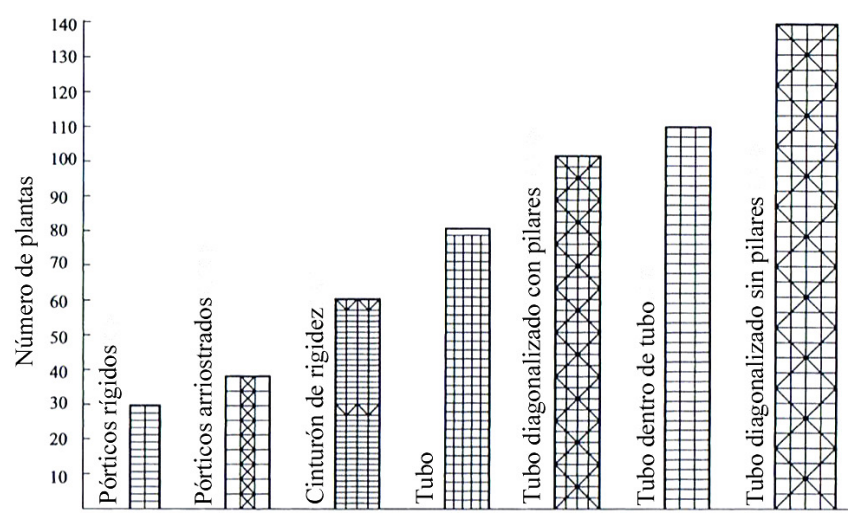

Figura 2. Clasificación de sistemas estructurales de acero y número de plantas de Fazlur R. Khan (11).

Pero es precisamente después de la segunda guerra mundial, y posteriormente en la época Icónica, cuando se potenció el concepto del edificio en altura siendo muchos los estudios con túnel de viento aerodinámico y Simulación Computacional Dinámica de Fluidos (Computational Fluid Dynamics, CFD) para estudiar el comportamiento del viento y su influencia sobre los edificios en altura, -Stathopoulos (12)- .

\subsection{Las mallas estructurales en arquitectura}

Como ya se ha indicado, las mallas estructurales envolventes de edificios en altura son una tipología muy utilizada en los últimos años. Las estructuras espaciales han tenido un 
gran impacto en la arquitectura, desde que alrededor del año 1806 se utilizó una malla como tipología estructural para edificación, con el diseño y construcción de la primera cúpula con esqueleto de hierro en París: la cúpula de la $\mathrm{Ha-}$ lle au Blé. Sin embargo, los primeros intentos para construir una malla espacial se le atribuyen al científico e inventor Alexander G. Bell hacia el año 1907, con sus inventos y demostraciones en el ámbito estructural de la ingeniería naval y aeronáutica. Otro referente es la cúpula geodésica del planetario de los talleres Carl Zeiss (1923). A pesar de ello, las mallas espaciales no tuvieron un papel relevante hasta los años 50 del siglo XX, cuando se aplicaron en el mundo arquitectónico, especialmente de la mano de Richard Buckminster Fuller que reinventó las cúpulas geodésicas -Eekhout (13)-.

Es en las últimas décadas cuando ha evolucionado una tipología estructural para los edificios en altura con sistemas estructurales Framed Tube o Tube-in-Tube, generando nuevas tendencias arquitectónicas. Edificios como el John Hancock y la Sears Tower en Chicago o la Hearst Tower en Nueva York, son algunos de los muchos ejemplos de edificios en altura con estructura metálica en la fachada.

Es por ello por lo que, de acuerdo a los puntos expuestos anteriormente, como el estudio de las líneas isostáticas para la optimización de estructuras así como la utilización de mallas espaciales, se realiza un estudio comparativo para optimizar mallas estructurales de acero envolventes de edificios en altura adaptando la geometría a la trayectoria de las líneas isostáticas del elemento resistente equivalente en función de sus solicitaciones.

\section{METODOLOGÍA}

A tenor de lo previamente enunciado, la metodología de trabajo del presente estudio comparativo para la optimización de mallas estructurales envolventes de edificios en altura, se establece de acuerdo a tres apartados. En el primero, se analizan las líneas isostáticas de cinco elementos verticales en voladizo (equivalentes a los edificios en altura), modelizados en dos dimensiones mediante elementos finitos para distintos estados de carga de acuerdo a la normativa posteriormente enunciada. Dicho estudio permite diseñar las posibles geometrías de comportamiento más eficiente, las cuales se presentan en el segundo apartado. En el tercer apartado se analizan los modelos convencionales y los posibles modelos optimizados, comparando sus resultados.

\subsection{Estudio de las líneas isostáticas}

Se estudian las líneas isostáticas, entendidas como las curvas envolventes de las tensiones principales, de cinco elementos estructurales en voladizo, para distintas esbelteces geométricas (H/B: 2, 3, 4, 5 y 6), para una base de $30 \mathrm{~m}$ (Figura 3), sometidos a distintas combinaciones de acciones, de acuerdo a la normativa utilizada, con el fin de determinar la trayectoria de las líneas isostáticas de cada elemento estructural para cada combinación de acciones.

Las trayectorias obtenidas para los distintos casos estudiados, se resumen en las Tablas 1 y 2, respectivamente en la parte superior y la base de los elementos analizados, de acuerdo a distintas combinaciones de acciones. Así como los puntos intermedios que se consideren necesarios.

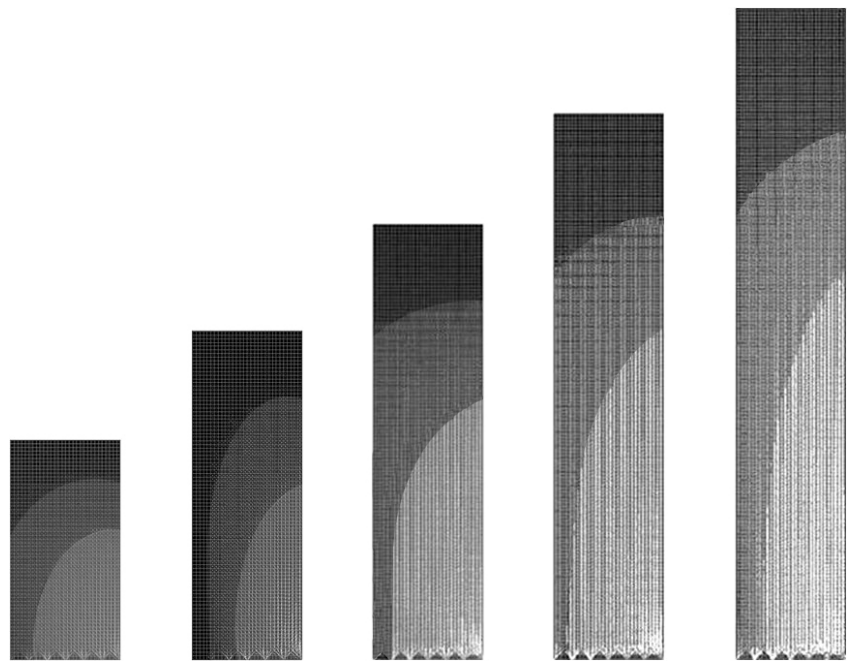

Figura 3. Estudio de las direcciones de las tensiones principales para cinco esbelteces geométricas.

Tabla 1. Inclinación ( $\mathrm{en}^{\circ}$ ), respecto la vertical, de las líneas isostáticas en la parte superior de los modelos.

\begin{tabular}{|c|c|c|c|c|c|}
\hline \multicolumn{5}{|c|}{ Inclinación $\left(\mathrm{en}^{\circ}\right)$ isostáticas en la parte superior de los modelos } \\
\hline Combin. & $\mathrm{H} / \mathrm{B}=2$ & $\mathrm{H} / \mathrm{B}=3$ & $\mathrm{H} / \mathrm{B}=4$ & $\mathrm{H} / \mathrm{B}=5$ & $\mathrm{H} / \mathrm{B}=6$ \\
\hline Comb. 1 & 15,80 & 16,12 & 16,44 & 16,77 & 17,12 \\
\hline Comb. 2 & 36,59 & 42,39 & 48,50 & 54,40 & 59,67 \\
\hline Comb. 3 & 69,39 & 73,11 & 75,84 & 78,10 & 80,28 \\
\hline
\end{tabular}

Tabla 2. Inclinación ( $\mathrm{en}^{\mathrm{o}}$ ), respecto la vertical, de las líneas isostáticas en la base de los modelos.

\begin{tabular}{|c|c|c|c|c|c|}
\hline \multicolumn{5}{|c|}{ Inclinación $\left(\mathrm{en}^{\mathrm{o}}\right.$ ) isostáticas en la base de los modelos } \\
\hline Combin. & $\mathrm{H} / \mathrm{B}=2$ & $\mathrm{H} / \mathrm{B}=3$ & $\mathrm{H} / \mathrm{B}=4$ & $\mathrm{H} / \mathrm{B}=5$ & $\mathrm{H} / \mathrm{B}=6$ \\
\hline Comb. 1 & 18,61 & 18,06 & 17,66 & 17,33 & 17,05 \\
\hline Comb. 2 & 19,06 & 18,20 & 17,57 & 17,08 & 16,69 \\
\hline Comb. 3 & 19,27 & 18,26 & 17,54 & 17,00 & 16,58 \\
\hline
\end{tabular}

Después de estudiar los resultados obtenidos para los cinco elementos analizados, se determina un trazado de las líneas isostáticas para elementos verticales en voladizo, que se utilizará para el diseño de las geometrías de las mallas propuestas en el apartado 2.2, adaptando las mallas convencionales uniformes en función de las líneas isostáticas, a la vez, que se atiende las necesidades arquitectónicas de los edificios, como es una distribución constante de las plantas en altura.

\subsection{Diseño de los modelos estructurales}

Una vez conocida la trayectoria de las líneas isostáticas, se adapta la forma de seis mallas para obtener las posibles geometrías envolventes optimizadas. Para el diseño de las mallas optimizadas se utilizan las triangulaciones más comunes para estructuras tipo celosía, -Torroja (14)-.

Dichas triangulaciones, permiten considerar la geometría de las mallas convencionales (Figura 4), adaptando la forma a la trayectoria de las líneas isostáticas definidas en el apartado previo 2.1. Asimismo, en la Figura 4, se muestran las geometrías de las seis posibles mallas envolventes optimizadas propuestas. Las mallas envolventes se diseñan para estructuras de entramado en tubo, por lo tanto, se utiliza 
Malla 1
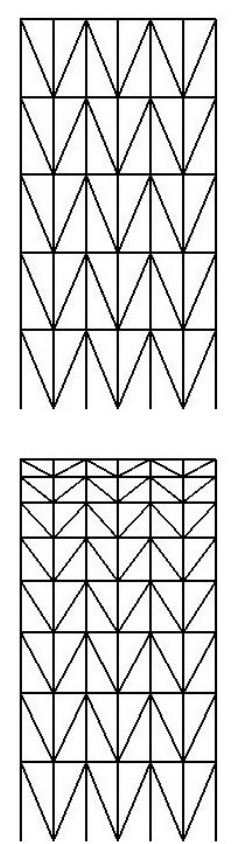

Malla 2
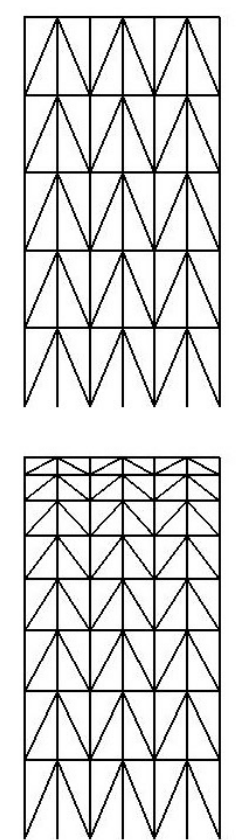

Malla 3
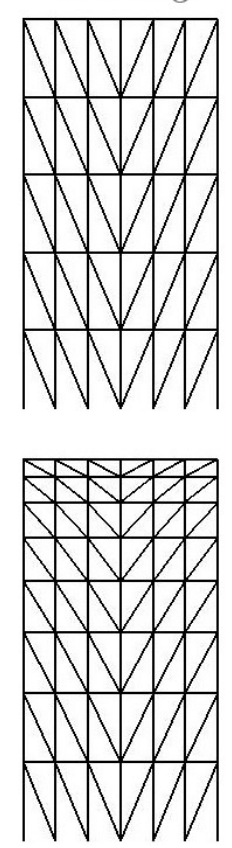

Malla 4
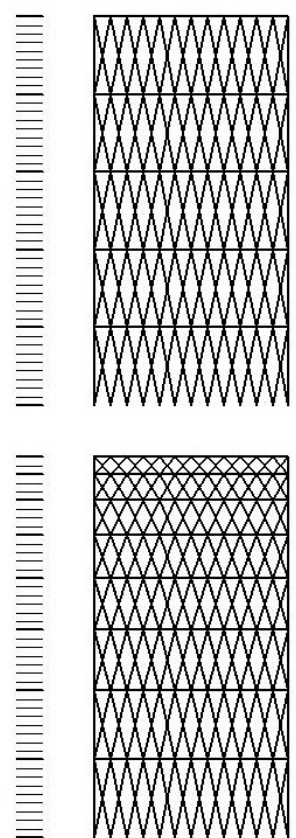

Malla 5
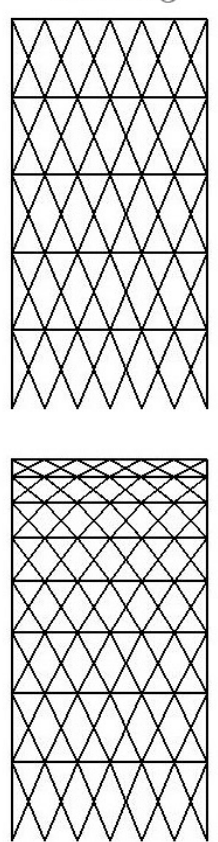

Malla 6
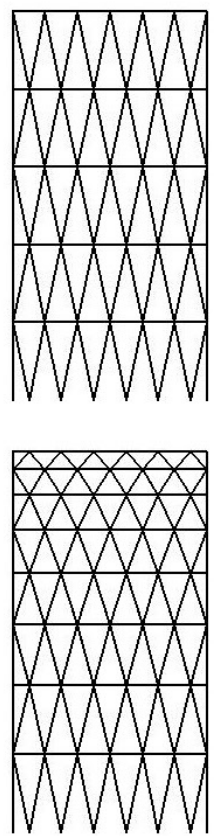

Figura 4. Geometrías (H/B: 2) de las seis mallas convencionales y geometrías de las posibles mallas optimizadas, teniendo en cuenta la trayectoria de las líneas isostáticas y el diseño arquitectónico.

la tipología estructural consistente en aprovechar las fachadas como elementos de contraviento. Este criterio se basa en que la estructura de entramado en tubo es un concepto estructural especialmente eficaz en edificios en altura, sometidos a flexión y torsión, principalmente en casos en que tal torsión se puede producir por la incidencia del viento en unas direcciones no coincidentes con la posición del centro resistente de las distintas plantas del edificio. La ventaja que tal solución presenta reside en que los elementos de contraviento se disponen a la máxima distancia respecto al centro de resistencia del edificio, aumentando así su inercia y su rigidez torsional.

Además de las características geométricas y mecánicas expuestas, los modelos diseñados de entramado en tubo para los edificios prismáticos proyectados, ofrecen un comportamiento estructural sencillo, atendiendo, de la mejor manera posible, las siguientes grandes ventajas que describe el Eurocódigo 8 (15) en su aspecto sismorresistente:

- Resistencia lateral.

- Simetría mecánica en planta.

- Regularidad, mecánica y geométrica, en altura, matizando la conveniente irregularidad decreciente de los intervalos de separación de los niveles principales horizontales de las mallas propuestas.

- Resistencia y rigidez a torsión.

- Ligereza, especialmente en la parte superior del edificio.

- Rigidez de los forjados en su plano, produciendo un beneficioso efecto diafragma.

Tal y como se ha expuesto, se consideran estructuras prismáticas (entramado en tubo) de base cuadrada y rectangular, para así dar cumplimiento a los puntos anteriormente descritos. En este sentido es adecuado mencionar las estructuras tubo de base circular, que no es objeto de estudio en el pre- sente comparativo ya que edificios con geometrías o cuerpos romos (no fuseladas o no aerodinámicas), de planta circular u otras curvas (formas ovaladas, elípticas, etc.), configuran una categoría que requiere un tratamiento específico.

Es conveniente realizar simulaciones con túnel de viento o túnel aerodinámico para determinar los coeficientes eólicos en cada zona de la superficie de la estructura y/o fachada.

\subsection{Análisis de las estructuras diseñadas}

En la última fase se analizan los modelos optimizados de acuerdo a la trayectoria de las líneas isostáticas y se comparan con los resultados de los modelos convencionales. El objetivo, de la Tesis y del presente estudio, es determinar y cuantificar si los modelos optimizados son más eficientes que los modelos convencionales, asimismo, establecer que mallas son más eficientes para determinadas alturas y, en consecuencia, para cada esbeltez geométrica considerada. Este apartado se desarrolla en profundidad en los puntos 3 y 4 que se describen a continuación.

\section{BASES DE CÁLCULO}

Para abordar el presente estudio comparativo, se analizan 600 modelos de edificios en altura de acuerdo a los criterios que se describen a continuación:

\subsection{Esbelteces de los edificios}

Se analizan cuatro alturas distintas de edificios, H: 45, 90, 135 y $180 \mathrm{~m}$. Para cada una de ellas se analizan tres esbelteces geométricas distintas de acuerdo a las siguientes dimensiones en planta, B: 15, 22,5 y $30 \mathrm{~m}$ de lado en el caso de base cuadrada, y de lado pequeño en el caso de base rectangular (de proporción 2 a 1). 


\subsection{Acciones consideradas}

Se han considerado las siguientes cargas, que se corresponden con una solución de estructura metálica:

- Peso propio de la estructura horizontal (perfiles/chapa colaborante): $3,50 \mathrm{kN} / \mathrm{m}^{2}$.

- Cargas permanentes de pavimentos, instalaciones y tabiquería: $2,20 \mathrm{kN} / \mathrm{m}^{2}$.

- Sobrecarga de uso residencial o de oficinas: 2,00-3,00 kN/m².

Las acciones del viento varían en el tiempo, por ello, las cargas producidas por el viento son cargas fluctuantes que producen presiones normales a la superficie de la estructura y/o a los distintos elementos de revestimiento, además de las fuerzas de rozamiento tangenciales a la superficie.

En edificios en los cuales cuya primera frecuencia propia sea elevada, es suficiente estimar de forma precisa las cargas adecuadamente promediadas en el tiempo, es decir, las «Cargas Estáticas Equivalentes», para así determinar las cargas generadas por el viento sobre la estructura y/o los revestimientos de los edificios analizados de acuerdo a las siguientes condiciones que establece la normativa utilizada, el Eurocódigo 1 (EC-1), parte 2-4 (16).

\subsection{Coeficientes de presión, de fuerzas y de momentos}

Para los casos que tengan una frecuencia natural de vibración elevada, por ejemplo valores superiores a $5 \mathrm{~Hz}$, se estimarán de forma precisa las cargas aerodinámicas adecuadamente promediadas en el tiempo, es decir, las «Cargas Estáticas Equivalentes».

En el caso de las estructuras tridimensionales estudiadas, los coeficientes aerodinámicos, $c$, de fuerza y de momento, se definen como el cociente entre la fuerza, $F$, o el momento, $M$, respectivamente y el producto de la presión dinámica, tal y como se muestra en las expresiones [1] y [2], -Holmes (10)-.

$$
\begin{gathered}
c_{F}=\frac{F}{\frac{1}{2} \rho_{a} U_{\infty}^{2} A_{\mathrm{ref}}} \\
c_{M}=\frac{M}{\frac{1}{2} \rho_{a} U_{\infty}^{2} C A_{\mathrm{ref}}}
\end{gathered}
$$

donde,

$c$, es el coeficiente aerodinámico;

$U$, es la velocidad de referencia (mapa eólico);

$\rho_{a}$, es la densidad del aire $\left(\rho_{a}=1,25 \mathrm{~kg} / \mathrm{m}^{3}\right)$;

$A_{\text {ref }}$, es la superficie de referencia (en cuerpos romos suele ser el área frontal del obstáculo);

$C$, es la longitud característica del obstáculo.

Las fuerzas de viento provocadas por presiones sobre las estructuras se determinan por medio de las fuerzas globales, que para las estructuras verticales en ménsula con una esbeltez superior a 2 y de sección prácticamente constante, como es el caso de los edificios en altura analizados, se concretan en la fuerza, $F_{\text {wj }}$, de acuerdo al EC-1, parte 2-4 (16).

El efecto de torsión debido a un viento oblicuo y/o no uniforme, se representa para las estructuras sensiblemente si- métricas no circulares, como una fuerza $F_{w}$, aplicada con una excentricidad mínima $e$, (respecto el centro resistente de la base), de acuerdo a la expresión [3].

$$
e=\frac{b}{10}
$$

donde,

$b$, es la dimensión perpendicular al eje principal considerado.

\subsection{Parámetros y modelización del viento}

La presión dinámica del viento se conoce como presión dinámica pico o de diseño, $q_{p}$, de acuerdo a la expresión [4] según el EC-1, parte 2-4 (16):

$$
q_{p}(z)=c_{e}(z) \cdot q_{\text {ref }}
$$

donde,

$q_{\text {ref }}$, es el coeficiente de presión dinámica básica o presión media de la velocidad de referencia que se describe en la expresión [5];

$c_{e}(z)$, es el coeficiente de exposición, el cual, contempla los efectos que, la rugosidad del terreno, la topografía y la altura sobre el nivel del suelo provocan sobre la velocidad del viento y la turbulencia.

$$
q_{\mathrm{ref}}=\frac{1}{2} \rho_{a} U_{b}^{2}
$$

donde,

$U_{b}$, es la velocidad básica o velocidad del viento de referencia según los mapas eólicos de Europa, (apartado 7.4, EC-1, parte 2-4 (16));

$\rho_{a}$, es la densidad del aire $\left(\rho_{a}=1,25 \mathrm{~kg} / \mathrm{m}^{3}\right)$

Suponiendo la carga del viento en ráfaga cuasi-estática, el coeficiente de exposición, $c_{e}(z)$, se establece de acuerdo al EC-1, parte 2-4 (16), para un coeficiente topográfico, $c_{t}=1$ (terreno llano) y considerando un tipo de zona I (mar abierto, lagos y campo abierto llano).

\subsection{Criterios de dimensionado y método}

Dado que los edificios analizados no superan los $200 \mathrm{~m}$ de altura y, por lo tanto, están dentro de la capa límite atmosférica o zona de influencia de la superficie terrestre, se calcularán las cargas de viento de acuerdo con el método simplificado del Eurocódigo 1 (16) para estructuras no susceptibles a la excitación dinámica. No obstante, cuando analizamos edificios en altura y por lo tanto bajo cargas de viento atmosférico, el flujo es turbulento (la velocidad del aire no es constante ni en el tiempo ni en el espacio), de forma que se considera la variación del viento con la altura, según la rugosidad del terreno.

Considerando que la presión media de una corriente fluctuante es mayor que la presión correspondiente a la velocidad media, es necesario definir el efecto de la turbulencia en las cargas estáticas de presión, de forma que se debe determinar la presión dinámica de cálculo en función de la rugosidad local y de la altura.

Este aspecto es muy importante, especialmente en las estructuras de mayor esbeltez (levemente dinámicas $\omega_{0}<5 \mathrm{~Hz}$ ). En 
este caso, se utilizará el método simplificado, pero será necesario aplicar el coeficiente dinámico, $c_{\mathrm{d}}$, dependiendo del tipo de estructura (hormigón, acero o mixta) y de la esbeltez del edificio. Dicho coeficiente dinámico, $c_{\mathrm{d}}$, tiene en cuenta la amplificación dinámica, y se multiplica a las «Cargas Estáticas Equivalentes» del método simplificado considerado. Los valores del coeficiente dinámico para estructura metálica se muestran en la Figura 5.

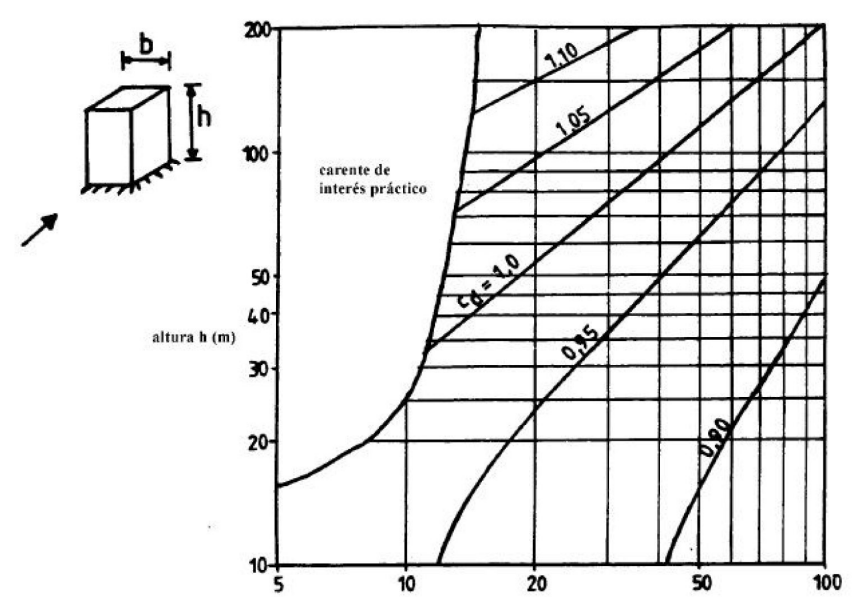

Figura 5. Coeficiente dinámico, $c_{\mathrm{d}}$, para edificios de estructura metálica, para una velocidad de referencia de $28 \mathrm{~m} / \mathrm{s}$, categoría del terreno I, $\delta_{a}=$ o y $\delta_{s}=0,0045 n_{1} \geq 0,05-\mathrm{EC}-1$, parte 2-4 (16)-.

La representación de la presión del viento de los edificios analizados, mediante el coeficiente dinámico, dependerá de la rigidez de éstos frente a la vibración resonante provocada por el viento. Para ello, será necesario conocer las frecuencias naturales y modos de vibración de los modelos estructurales estudiados durante el proceso de vibración libre.

En ningún caso se analizan edificios con una esbeltez y una tipología estructural para los cuales el coeficiente dinámico, $c_{\mathrm{d}}$, supere el valor 1,2. De la misma manera que tampoco se realizarán estudios con edificios con una frecuencia natural inferior a $1 \mathrm{~Hz}$, donde la respuesta a resonancia es significativa, -Holmes (10)-. En caso contrario, se debería aplicar el método detallado para calcular las cargas de viento. No son tampoco objeto del estudio las estructuras susceptibles de excitación. En este mismo sentido, no se tienen en cuenta el desprendimiento de remolinos y el galope transversal (fenómenos aerodinámicos) en los edificios analizados, es decir, respectivamente las cuestiones relativas a las fuerzas laterales periódicas que causan vibraciones laterales, -Meseguer, et al. (17)-, y la inestabilidad de frecuencia baja producida por la oscilación lateral de la estructura, -Meseguer, et al. (18)-.

Los coeficientes eólicos en edificios en altura, prismáticos y de planta rectangular, de acuerdo al Eurocódigo 1, parte 2-4 (19), se muestran en la Tabla 3:

Tabla 3. Coeficientes eólicos en edificios prismáticos de planta rectangular según EC-1, parte 2-4 (19).

\begin{tabular}{|l|c|c|c|c|}
\cline { 2 - 5 } \multicolumn{1}{c|}{} & \multicolumn{4}{c|}{$\begin{array}{c}\text { Esbeltez menor planos vertical } \\
\text { y horizontal paralelos al viento }\left(\mathbf{z}_{e} / \boldsymbol{d}\right)\end{array}$} \\
\cline { 2 - 5 } & $\mathbf{> 4 , 0}$ & $\mathbf{2 , o}$ & $\mathbf{1 , 3 3}$ & $\leq \mathbf{1 , 0}$ \\
\hline $\mathrm{C}_{\mathrm{p}}$ (presión) & 0,60 & 0,73 & 0,78 & 0,80 \\
\hline $\mathrm{C}_{\mathrm{s}}$ (succión) & $-0,3$ & $-0,3$ & $-0,3$ & $-0,3$ \\
\hline
\end{tabular}

donde,

$d$, es la base del edificio en el plano paralelo al viento; $z_{e}$, es la altura de referencia, la menor entre $h$ (altura del edificio) y $b$ (base del edificio en el plano perpendicular al viento).

\subsection{Combinaciones de acciones}

De acuerdo al Código Técnico de la Edificación, CTE (20), para Estado Límite de Servicio (ELS), se toman las siguientes combinaciones de acciones:

- Combinación característica. Se analizarán los efectos producidos por las acciones de corta duración y que puedan resultar irreversibles, de acuerdo a la expresión [6].

$$
\sum_{j \geq 1} G_{k, j}+P+Q_{k, 1}+\sum_{i \geq 1} \Psi_{0, i} \cdot Q_{k, i}
$$

- Combinación frecuente. Se analizarán los efectos producidos por las acciones de corta duración y que pueden resultar reversibles, de acuerdo a la expresión [7].

$$
\sum_{j \geq 1} G_{k, j}+P+\Psi_{1,1} \cdot Q_{k, 1}+\sum_{i \geq 1} \Psi_{2, i} \cdot Q_{k, i}
$$

- Combinación casi permanente. Se analizarán los efectos producidos por las acciones de larga duración, de acuerdo a la expresión [8].

$$
\sum_{j \geq 1} G_{k, j}+P+\sum_{i \geq 1} \Psi_{2, i} \cdot Q_{k, i}
$$

\subsection{Limitaciones de deformaciones}

Al tratarse de un estudio sobre el comportamiento global de la estructura frente a fuerzas horizontales, el parámetro analizado ha sido el desplazamiento horizontal en la parte superior del edificio bajo el efecto de dichas cargas. Atendiendo a las limitaciones especificadas en el Código Técnico de la Edificación, CTE (20) y las combinaciones a emplear, se puede concretar una tabla del siguiente tipo, (Tabla 4):

Tabla 4. Limitaciones de desplome según CTE (20).

\begin{tabular}{|l|c|c|c|}
\hline & Combinación & $\begin{array}{c}\text { Desplome } \\
\text { total }\end{array}$ & $\begin{array}{c}\text { Desplome } \\
\text { local }\end{array}$ \\
\hline $\begin{array}{l}\text { Integridad } \\
\text { elementos }\end{array}$ & Característica & $1 / 500$ & $1 / 250$ \\
\hline $\begin{array}{l}\text { Apariencia } \\
\text { edificio }\end{array}$ & Casi permanente & - & $1 / 250$ \\
\hline
\end{tabular}

\subsection{Perfiles utilizados}

Los perfiles utilizados para las mallas analizadas son perfiles de sección cuadrada de la serie europea de perfiles, eligiendo aquel de menor peso que cumpla con las condiciones de dimensionado correcto y que da una respuesta satisfactoria al cumplimiento de la normativa para ELS.

\section{ANÁLISIS Y RESULTADOS}

Cuando analizamos modelos estructurales de edificios en altura, es fundamental tener en cuenta los efectos dinámicos del viento, ya que esta tipología de edificio es muy sensible para este tipo de carga. Bajo esta acción, los edificios esbeltos se exponen a cargas dinámicas en la dirección del viento 
y en la dirección perpendicular a él, considerando asimismo, los movimientos torsionales tal y como se muestra en la Figura 6.

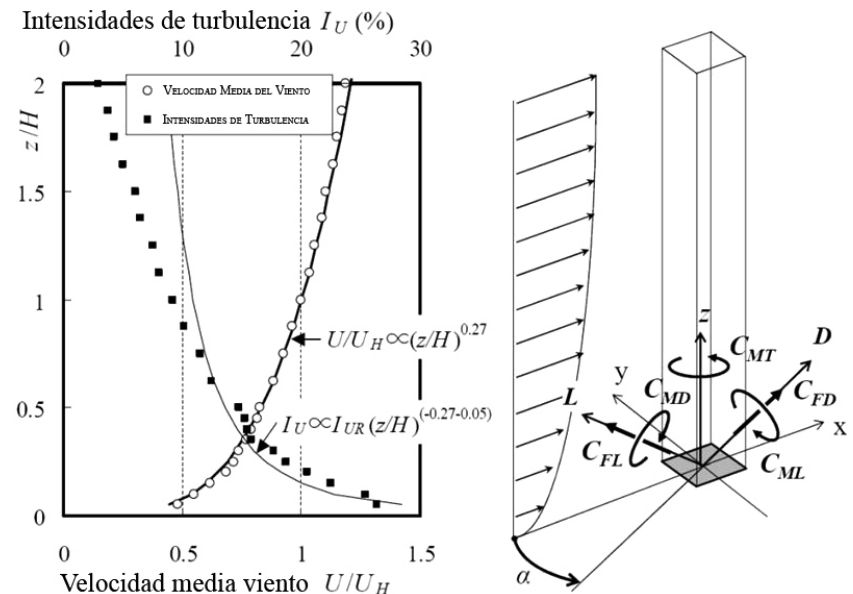

Figura 6. Fuerzas, momentos y turbulencias resultantes del viento, -Tamura, et al. (21)-.

Las cargas de viento se han determinado mediante el método simplificado de «Cargas Estáticas Equivalentes», y aplicando el coeficiente dinámico en los casos que así lo han requerido de acuerdo a las condiciones descritas en el apartado 3.5. Las frecuencias y modos de vibración de las estructuras se deben calcular para determinar la rigidez de las estructuras para evitar la vibración resonante provocada por el viento. Este punto es fundamental, dado que es necesario para determinar las cargas de viento mediante el método simplificado en aquellas estructuras cuyas propiedades estructurales no las hacen susceptibles a la excitación dinámica. En las estructuras levemente dinámicas, se utilizará adicionalmente el coeficiente dinámico, $c_{\mathrm{d}}$.

De acuerdo a las bases de cálculo, se analizan 600 modelos, de los cuales 300 corresponden a modelos con geometrías de mallas uniformes convencionales y los 300 restantes a mallas de las posibles geometrías optimizadas según el apartado 2.2.

\subsection{Análisis multimodal no lineal (P-delta)}

El análisis de los distintos modelos estructurales se realizará con un análisis multimodal no lineal ( $P$-delta). Esto se debe a que se analiza la no linealidad geométrica de los modelos analizados, tal y como especifica la normativa, considerando así los efectos de segundo orden derivados de las deformaciones que pueden aparecer bajo cargas muertas y variables que van a provocar desplazamientos a tener en cuenta en la coronación de los edificios en altura. Para ello, se realiza el análisis de los modelos estudiados con el programa de cálculo SAP 2000, que permite todas las opciones de cálculo anteriormente descritas.

El análisis multimodal permite determinar las frecuencias naturales y los modos de vibración de la estructura durante el proceso de vibración libre. Para el caso de un estudio de los problemas de un material elástico que se rige por las leyes de elasticidad de Hooke, la ecuación generalizada de movimiento escrita de forma conjunta responde, en forma matricial, a la expresión [9].

$$
\mathbf{M} \ddot{\mathbf{x}}(t)+\mathbf{C} \dot{\mathbf{x}}(t)+\mathbf{K x}(t)=\mathbf{f}(t)
$$

donde,

$\mathbf{M}, \mathbf{C}, \mathbf{K}$, son respectivamente las matrices de masa, de amortiguamiento y de rigidez de la estructura, y, f, la fuerza dinámica. $\ddot{\mathbf{x}}, \dot{\mathbf{x}}, \mathbf{x}$, son los vectores que representan respectivamente la posición, velocidad y aceleración de un conjunto de puntos de la estructura.

\subsection{Evaluación del rendimiento}

Para realizar el comparativo entre los modelos convencionales y los modelos optimizados, es necesario determinar el rendimiento estructural, $R$, de cada estructura analizada de acuerdo a la expresión [10] formulada considerando el concepto expuesto por Estévez, et al. (22) (23), que determina el rendimiento global de una estructura de acuerdo a las deformaciones máximas y el peso propio de la estructura, para un determinado estado de cargas $(Q)$ y una altura $(H)$.

$$
R_{e}=\frac{Q \cdot H}{\delta \cdot P_{e}}
$$

donde,

$Q$, es la carga total soportada;

$H$, altura del edificio estudiado;

$\delta$, desplazamiento horizontal máximo en la coronación del edificio;

$P_{e}$, es el peso propio de la estructura.

Este valor del rendimiento estructural se optimizará cuando el producto, $\delta \cdot P_{e}$, sea menor.

\subsection{Comparación resultados}

A continuación, se comparan los resultados de rendimiento estructural, $R_{e}$, de los seis modelos de mallas convencionales con los seis modelos de mallas optimizadas, de acuerdo a las distintas esbelteces de los edificios definidas en el apartado 3.1.

Se muestran los resultados de mayor relevancia de los dos comparativos. El primero se refiere a los resultados de los modelos de base cuadrada y el segundo muestra los resultados de base rectangular en la dirección de mayor esbeltez.

\subsubsection{Resultados comparativos: modelos de base cuadrada}

En la Figura 7 se muestran seis de los modelos analizados de base cuadrada (tres de malla convencional y tres de malla optimizada).

En la Tabla 5, se comparan los resultados (peso estructura) para una altura de $45 \mathrm{~m}$ y un mismo desplazamiento en la coronación. Las mallas optimizadas se indican con *. El resto de resultados se comparan en la Figura 8 (gráficos).

4.3.2. Resultados comparativos: modelos de base rectangular (proporción 2 a 1)

En la Figura 9 se muestran seis de los modelos analizados de base rectangular (tres de malla convencional y tres de malla optimizada).

En la Tabla 6, se comparan los resultados (peso estructura) para una altura de $45 \mathrm{~m}$ y un mismo desplazamiento en la coronación. Las mallas optimizadas se indican con *. El resto de resultados se comparan en la Figura 10 (gráficos). 

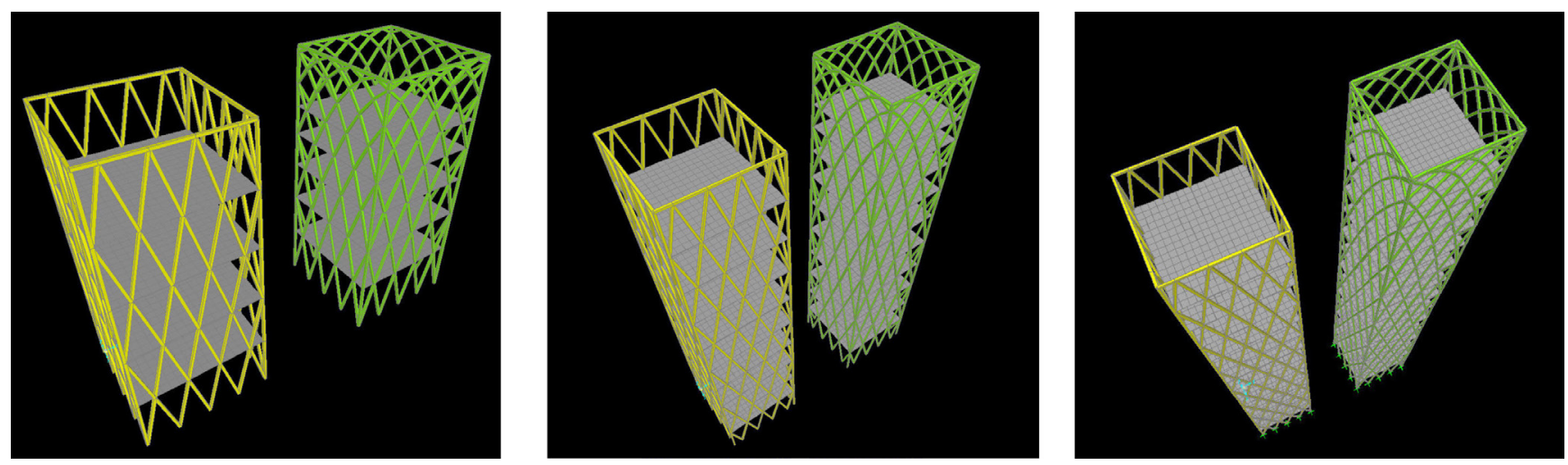

Figura 7. Imágenes tomadas de los modelos de malla 6 y 6* de base cuadrada analizados con SAP 2000.

Tabla 5. Comparativo de resultados de mallas convencionales y mallas optimizadas ( ${ }^{*}$ ). Base cuadrada de $22,5 \mathrm{~m}$ y para una altura de $45 \mathrm{~m}(\mathrm{H} / \mathrm{B}: 2)$.

\begin{tabular}{|c|c|c|c|c|c|c|c|c|}
\hline Malla 1 & H: $45,0 \mathrm{~m}$ & Malla 1* & Malla 2 & $\mathrm{H}: 45, \mathrm{O} \mathrm{m}$ & Malla 2* & Malla 3 & H: $45,0 \mathrm{~m}$ & Malla $3^{*}$ \\
\hline $9,0 \mathrm{~cm}$ & $\delta_{\max }$ & $9,0 \mathrm{~cm}$ & $9,0 \mathrm{~cm}$ & $\delta_{\text {max }}$ & $9,0 \mathrm{~cm}$ & $9,0 \mathrm{~cm}$ & $\delta_{\max }$ & $9,0 \mathrm{~cm}$ \\
\hline $1614,9 \mathrm{kN}$ & $78,15 \%$ & $1262,1 \mathrm{kN}$ & $2230,3 \mathrm{kN}$ & $70,18 \%$ & $1565,2 \mathrm{kN}$ & $1198,8 \mathrm{kN}$ & $83,65 \%$ & $1002,8 \mathrm{kN}$ \\
\hline Malla 4 & $\mathrm{H}: 45, \mathrm{O} \mathrm{m}$ & Malla $4 *$ & Malla 5 & H: $45,0 \mathrm{~m}$ & Malla $5^{*}$ & Malla 6 & H: $45,0 \mathrm{~m}$ & Malla 6* \\
\hline $9,0 \mathrm{~cm}$ & $\delta_{\text {max }}$ & $9,0 \mathrm{~cm}$ & $9,0 \mathrm{~cm}$ & $\delta_{\text {max }}$ & $9,0 \mathrm{~cm}$ & $9,0 \mathrm{~cm}$ & $\delta_{\text {max }}$ & $9,0 \mathrm{~cm}$ \\
\hline $1330,9 \mathrm{kN}$ & $74,71 \%$ & $994,4 \mathrm{kN}$ & $654,4 \mathrm{kN}$ & $88,13 \%$ & $576,7 \mathrm{kN}$ & $1196,9 \mathrm{kN}$ & $74,95 \%$ & $897,1 \mathrm{kN}$ \\
\hline
\end{tabular}
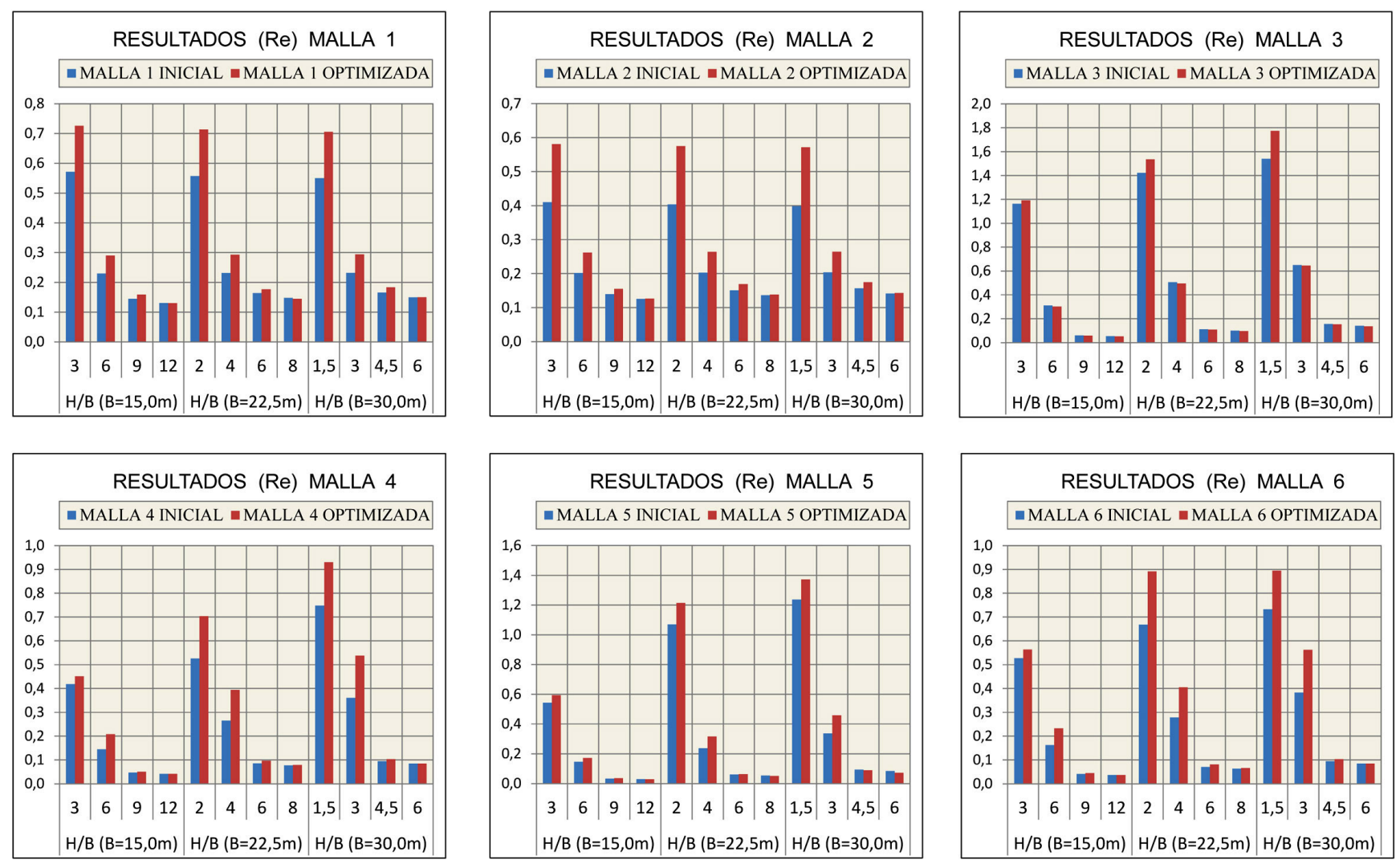

Figura 8. Comparativo del rendimiento de los 12 modelos de base cuadrada (seis mallas iniciales o convencionales y seis mallas optimizadas*) para las distintas esbelteces geométricas analizadas. 

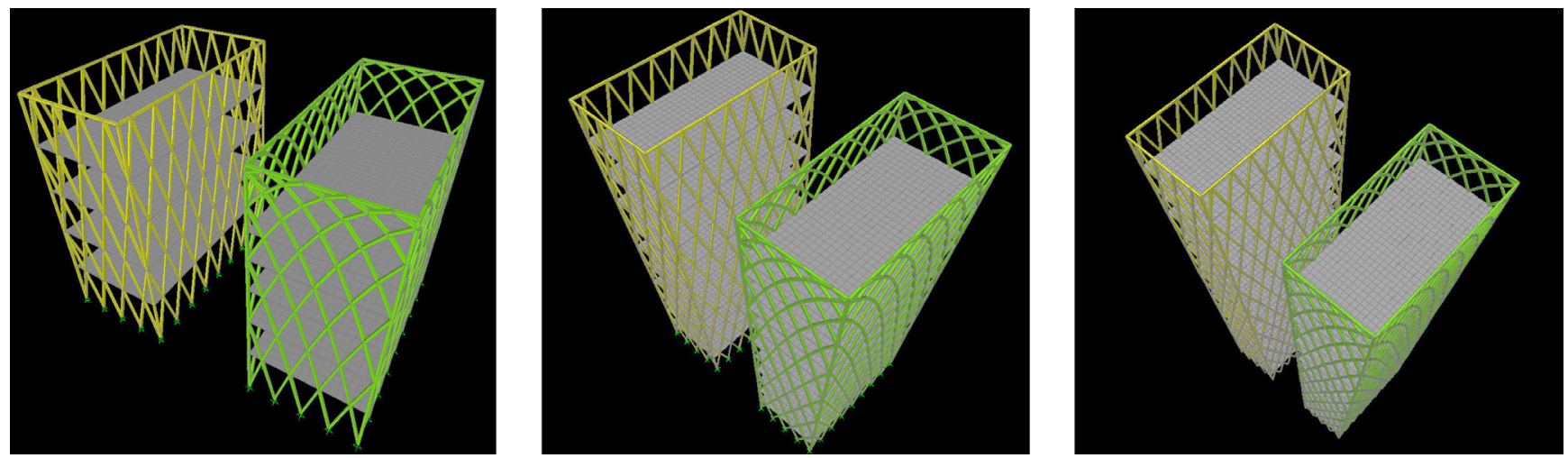

Figura 9. Imágenes tomadas de los modelos de malla 6 y 6* de base rectangular analizados con SAP 2000.

Tabla 6. Comparativo de resultados de mallas convencionales y mallas optimizadas ${ }^{*}$ ). Base rectangular de $22,5 \mathrm{~m}$ de lado pequeño y una altura de $45 \mathrm{~m}$ (H/B: 2).

\begin{tabular}{|c|c|c|c|c|c|c|c|c|}
\hline Malla 1 & H: $45,0 \mathrm{~m}$ & Malla 1* & Malla 2 & H: $45,0 \mathrm{~m}$ & Malla 2* & Malla 3 & H: $45,0 \mathrm{~m}$ & Malla $3^{*}$ \\
\hline $9,0 \mathrm{~cm}$ & $\delta_{\max }$ & $9,0 \mathrm{~cm}$ & $9,0 \mathrm{~cm}$ & $\delta_{\max }$ & $9,0 \mathrm{~cm}$ & $9,0 \mathrm{~cm}$ & $\delta_{\text {max }}$ & $9,0 \mathrm{~cm}$ \\
\hline $2044,5 \mathrm{kN}$ & $85,79 \%$ & $1754, \mathrm{o} \mathrm{kN}$ & $3032,8 \mathrm{kN}$ & $76,17 \%$ & $2310,1 \mathrm{kN}$ & $1938,8 \mathrm{kN}$ & $93,84 \%$ & $1819,4 \mathrm{kN}$ \\
\hline Malla 4 & $\mathrm{H}: 45, \mathrm{O} \mathrm{m}$ & Malla 4* & Malla 5 & $\mathrm{H}: 45, \mathrm{O} \mathrm{m}$ & Malla $5^{*}$ & Malla 6 & $\mathrm{H}: 45, \mathrm{O} \mathrm{m}$ & Malla 6* \\
\hline $9,0 \mathrm{~cm}$ & $\delta_{\text {max }}$ & $9,0 \mathrm{~cm}$ & $9,0 \mathrm{~cm}$ & $\delta_{\text {max }}$ & $9,0 \mathrm{~cm}$ & $9,0 \mathrm{~cm}$ & $\delta_{\text {max }}$ & $9,0 \mathrm{~cm}$ \\
\hline $2664,8 \mathrm{kN}$ & $93,26 \%$ & $2485,3 \mathrm{kN}$ & $1703,6 \mathrm{kN}$ & $91,84 \%$ & $1564,6 \mathrm{kN}$ & $1638,8 \mathrm{kN}$ & $82,61 \%$ & $1353,9 \mathrm{kN}$ \\
\hline
\end{tabular}
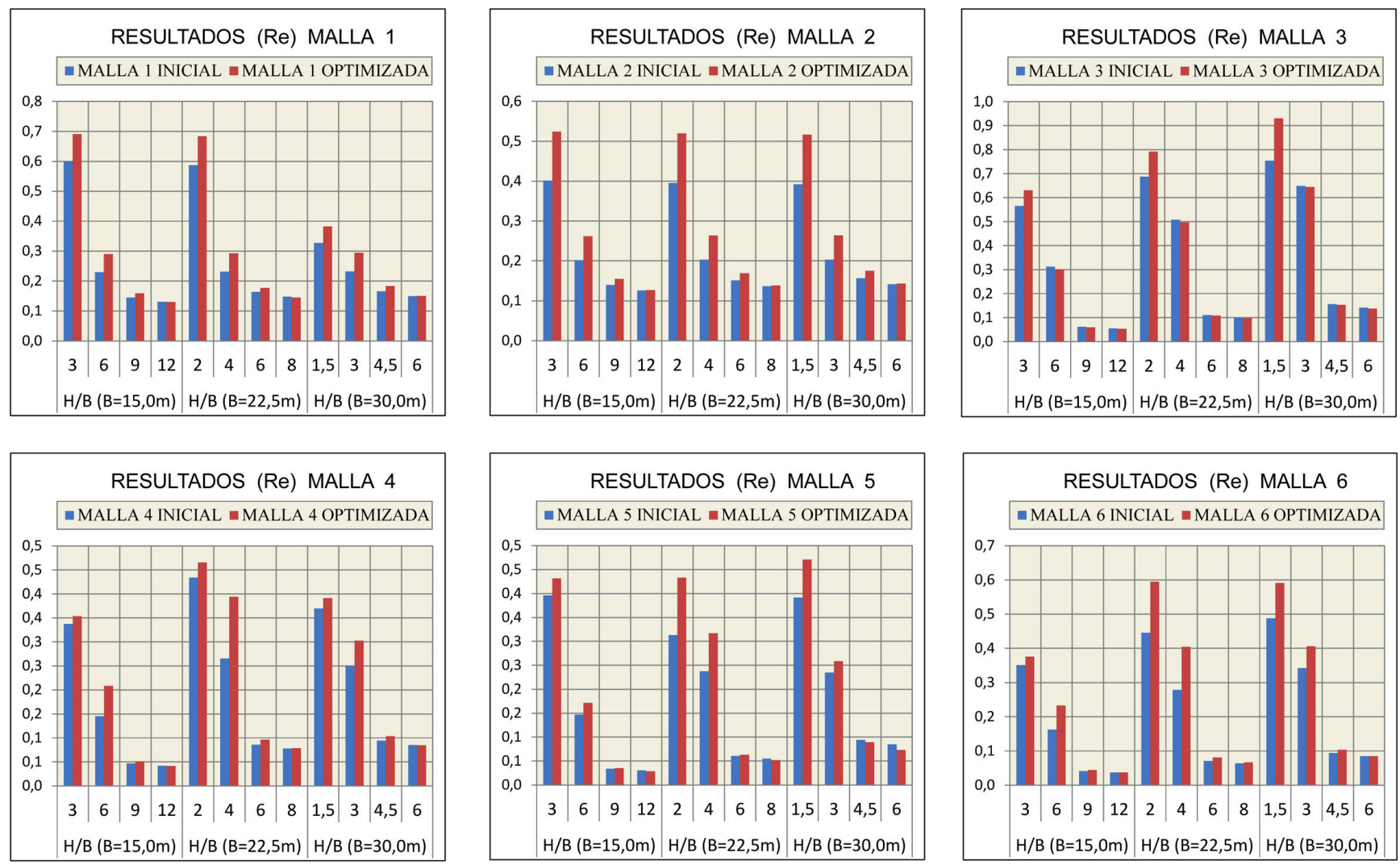

Figura 10. Comparativo del rendimiento de los 12 modelos de base rectangular (seis mallas iniciales o convencionales y seis mallas optimizadas*) para las distintas esbelteces geométricas analizadas. 


\section{CONCLUSIONES}

De acuerdo a los resultados anteriormente presentados, se observa que en general todos los modelos de mallas optimizadas presentan mejores resultados de rendimiento que las geometrías de mallas convencionales, tal y como se especifica en los siguientes cinco párrafos.

a) Con el presente estudio comparativo de mallas espaciales de acero envolventes de edificios en altura (dentro de un orden máximo analizado de $180 \mathrm{~m}$ ), se demuestra que, para determinadas alturas o esbelteces geométricas, las tipologías de mallas propuestas son más eficientes cuando adoptan la trayectoria de las líneas isostáticas de acuerdo a sus solicitaciones.

b) Es conveniente destacar que la optimización propuesta depende más de la geometría de las mallas analizadas que del hecho de que se optimice su diseño. En consecuencia, hay mallas más adecuadas para determinadas alturas o esbelteces como se puede observar en los resultados de las mallas 1, 2, 4 y 6. En las mallas 3 y 5, debido a sus características geométricas, la ganancia en eficiencia es significativamente menor a los resultados obtenidos en las otras tipologías, que presentan resultados más satisfactorios. c) Se observa que para alturas próximas a los $135 \mathrm{~m}$, la tendencia de optimización converge, siendo cada vez más pequeña la diferencia de rendimiento estructural entre las mallas convencionales y las mallas optimizadas. Aun así, se analizan los edificios de $180 \mathrm{~m}$ de altura con el fin de certificar la tendencia preponderante de este estudio. Se obtiene que para los edificios próximos a los $180 \mathrm{~m}$ el rendimiento estructural es prácticamente igual, no existiendo una optimización significativa.

d) Los modelos de base rectangular presentan resultados muy parecidos en ambas direcciones (difiriendo del orden del $5 \%$ ), pero en ambos casos, los resultados de optimización (comparativo de los modelos convencionales con los modelos optimizados) son ligeramente de carácter inferior que los resultados obtenidos en el comparativo de estructuras de base cuadrada.

e) A partir de este estudio se puede considerar que el análisis realizado para optimizar mallas envolventes de edificios en altura es factible, atendiendo a la relevancia de sus resultados de acuerdo a los criterios establecidos, considerando la viabilidad de análisis de edificios distintos en situaciones de mayor complejidad para aquellas geometrías más eficientes.

\section{REFERENCIAS}

(1) Fernández, J.A., Navarro, J.R. (1999). Eduardo Torroja. Ingeniero. Madrid: Pronaos.

(2) Thompson, D’A. (1961). Sobre el crecimiento y la forma (1 ${ }^{\text {st }}$ edition). Tyler-Bonner, J. (Ed.). United Kingdom: Cambridge University Press.

(3) Gordon, J.E. (1978). Structures or Why things don't fall down (1 $1^{\text {st }}$ edition). United Kingdom: Penguin Books Ltd. Doi: http://dx.doi.org/10.1007/978-1-4615-9074-3.

(4) Salvadori, M., Heller, R. (1998). Estructuras para arquitectos ( $3^{\text {a }}$ edición). Madrid: Kliczkowski Publisher.

(5) Moussavi, F. (2009). The Function of Form. Actar and the Harvard University Graduate School of Design.

(6) Muttoni, A. (2011). The Art of Structures. Laussane: EPFL Press.

(7) Mimram, M. (1983). Étude appliquée à l'œuvre de Robert Le Ricolais. París: Bordas.

(8) Larson, G.R. (2011). The Great Builders. William Le Baron Jenney. Developed Chicago's distinctive skyscrapers. Powell, K. (Ed.). London: Thames \& Hudson, Ltd.

(9) Gómez-Hermoso, J., et al. (2013). Proyecto de edificios altos. Monografía 20/21 ACHE (Asociación Científico-Técnica del Hormigón Estructural). Madrid: Fiselgraf.

(10) Holmes, J.D. (2007). Wind loading of structures ( $2^{\text {nd }}$ edition). London and New York: Taylor \& Francis Group.

(11) Khan, F.R. (1969). Recent structural systems in steel for high-rise buildings. En Proceedings of the British Constructional Steelwork Association Conference on Steel in Architecture, Londres.

(12) Stathopoulos, T. (2002). The Numerical Wind Tunnel for Industrial Aerodynamics: Real or Virtual in the New Millenium? Journal of Wind \& Structures, 5(2-4): 193-208, doi: http://dx.doi.org/10.1016/So167-6105(01)00206-9.

(13) Eekhout, M. (1989). Architecture in Space Structures. Rotterdam: Uitgeverij o1o Publishers.

(14) Torroja, E. (2010). Razón y ser de los tipos estructurales (Edición revisada por Torroja, J.A.). Madrid: Consejo Superior de Investigaciones Científicas / Ediciones Doce Calles, S.L.

(15) AEN/CTN 140-Eurocódigos Estructurales. (1998). UNE-ENV 1991-1-1. Eurocódigo 8: Disposiciones para el proyecto de estructuras sismorresistentes. Parte 1-1: Reglas generales. Acciones sísmicas y requisitos generales de las estructuras. AENOR, Madrid.

(16) AEN/CTN 140-Eurocódigos Estructurales. (1998). UNE-ENV 1991-2-4. Eurocódigo 1: Bases de proyecto y acciones en estructuras. Parte 2-4: Acciones en estructuras. Acciones del viento. AENOR.

(17) Meseguer, J., Sanz-Andrés, A. (2010). Aerodinámica básica (2 ${ }^{\mathrm{a}}$ Edición). Madrid: Garceta Grupo Editorial.

(18) Meseguer, J., Sanz-Andrés, A., Pindado, S., Franchini, S., Alonso, G. (2013). Aerodinámica civil. Efectos del viento en edificaciones y estructuras ( $2^{\text {a }}$ Edición). Madrid: Garceta Grupo Editorial.

(19) AEN/CTN 140-Eurocódigos Estructurales. (1998). UNE-ENV 1991-2-4. Eurocódigo 1: Bases de proyecto y acciones en estructuras. Parte 2-4: Acciones en estructuras. Acciones del viento, 10.2: Coeficientes aerodinámicos en edificios. AENOR.

(20) CTE. (2006). Documento Básico SE-AE (Seguridad Estructural: Bases de Cálculo y Acciones en la edificación). Madrid: Código Técnico de la Edificación. 
(21) Tamura, Y., Kim, Y., Tanaka, H., Ohtake, K., Nakai, M. (2010). Aerodynamics characteristics of tall building models with various unconventional configurations. En Structures Congress 2010 (pp. 3104-3113).

(22) Estévez, J., Martín, E. (2002). Influencia de los parámetros de diseño en el rendimiento estructural de vigas de celosía. Informes de la Construcción, 54(479): 17-28, doi: http://dx.doi.org/10.3989/ic.2002.v54.i479.617.

(23) Estévez, J., Martín, E. (2003). Análisis del rendimiento estructural de vigas Vierendeel. Informes de la Construcción, 54(483): 27-37, doi: http://dx.doi.org/10.3989/ic.2003.v54.i483.573. 